Supporting information for

\title{
Lung Targeting Lysostaphin Microspheres for Methicillin-Resistant Staphylococcus aureus Pneumonia Treatment and Prevention
}

\author{
Xiuhui Lin, Jian He, Wanlin Li, Yuchen Qi, Huiqun Hu, Dongxiao Zhang, Feng Xu*, \\ Xiaoyuan Chen*, Min Zhou*
}

*Corresponding authors. E-mail: zhoum@zju.edu.cn (Min Zhou); chen.shawn@nus.edu.sg (Xiaoyuan Chen); xufeng99@zju.edu.cn (Feng Xu)

This file includes:

\section{METHODS}

\section{REFERENCES}

Supporting Figure 1. Design and characterization of hollow visualized LyIR@MS

Supporting Figure 2. Minimal inhibition concentration of LyIR@MS against MRSA

Supporting Figure 3. Anti-biofilm activity of LyIR@MS in a time-dependent manner

Supporting Figure 4. Confocal laser scanning microscopy images of lung tissues at different times after intravenous injection of cy5.5-staining LyIR@MS

Supporting Figure 5. Bacterial load in the lung of mice in the post-exposure treatment study

Supporting Figure 6. Analysis of cellular composition in BALF $24 \mathrm{~h}$ after infection

Supporting Figure 7. H\&E and immunohistochemical staining of lung sections in the postexposure treatment study

Supporting Figure 8. Bacterial load in the lung of mice in pre-exposure prophylaxis study 


\section{METHODS}

\section{Bactericidal Activity against Planktonic Cells}

Ly and LyIR@MS (31.25 $\mu \mathrm{g} / \mathrm{mL}$ or $125 \mu \mathrm{g} / \mathrm{mL}$ of lysostaphin) in $1 \mathrm{~mL}$ TSB was added to 15 $\mathrm{mL}$ round-bottom tubes, along with mid-logarithm growth-phase USA300 cultures at $1 \times 10^{7}$ CFU/mL in $1 \mathrm{~mL}$ TSB, followed by aerobically incubation at $37^{\circ} \mathrm{C}$ at 250 r.p.m. The bactericidal activity of TSB alone and MS alone were also investigated. We collected aliquots of $100 \mu \mathrm{L}$ of the mixed suspension at $0,0.25,3,6,9,12$, and $24 \mathrm{~h}$ after incubation. a 10 -fold serial dilution was prepared. The bactericidal activity was determined by plating $100 \mu \mathrm{L}$ of appropriate dilutions on TSA plates. The colony-forming units (CFU) of different treatments were enumerated after $24 \mathrm{~h}$ culture and expressed as $\log _{10} \mathrm{CFU} / \mathrm{mL}$.

\section{Live/Dead Bacterial Viability Assay}

$1 \times 10^{7} \mathrm{CFU} / \mathrm{mL}$ bacterial culture in TSB was prepared as above and cocultured with TSB (Control), MS, Ly, and LyIR@MS at a final concentration of $125 \mu \mathrm{g} / \mathrm{mL}$. The bacteria were collected by centrifugation at 5,000 r.p.m for 5 min after $3 \mathrm{~h}$ incubation with the different treatments, washed twice with PBS. The pellet was re-suspended and stained with $10 \mu \mathrm{M}$ PI (red-fluorescent nucleic acid stain propidium iodide) and SYTO 9 (SYTO 9 green-fluorescent nucleic acid stain) solution for $20 \mathrm{~min}$. The stained bacteria were centrifugated and washed twice with PBS, and then were imaged with TCS SP8 Leica confocal microscopy.

\section{Transmission Electron Microscopy (TEM)}

A stationary growth-phase culture of MRSA at $1 \times 10^{7} \mathrm{CFU} / \mathrm{mL}$ in $1 \mathrm{~mL}$ TSB was exposed to a lethal dose of LyIR@MS to a final volume of $2 \mathrm{~mL}$. TSB, MS alone, and Ly served as the controls. Then the bacteria with different treatments were incubated at $37^{\circ} \mathrm{C}$ at 250 r.p.m for $3 \mathrm{~h}$. The bacteria were collected by centrifugation at 5,000 r.p.m for $5 \mathrm{~min}$, washed with PBS, and fixed with $2.5 \%$ glutaraldehyde at $4{ }^{\circ} \mathrm{C}$ overnight. Fixed bacteria were processed according to a standard TEM procedure. Briefly, the procedure includes double fixation, dehydration, infiltration, embedding, ultrathin sectioning, staining. At last, the stained ultrathin sections were observed in Hitachi Model H-7650 TEM. 


\section{Serial Passage Experiments}

MRSA at $1 \times 10^{7} \mathrm{CFU} / \mathrm{mL}$ in $100 \mu \mathrm{L}$ TSB was seeded to each well of a 96-well plate and incubated at $37^{\circ} \mathrm{C}$ statically for $48 \mathrm{~h}$ to form biofilms. Then the planktonic bacteria were washed twice with PBS and $100 \mu \mathrm{L}$ fresh TSB containing different concentrations of LyIR@MS or TSB alone was added to the well and incubated at $37^{\circ} \mathrm{C}$ again. After treatment of $24 \mathrm{~h}$, the biofilm was washed with PBS and then the bacteria embedded in the biofilm was resuspended in $200 \mu \mathrm{L}$ TSB, then transferred to a $15 \mathrm{~mL}$ round-bottom tube for $24 \mathrm{~h}$ incubation. For the serial passage experiments, fresh cultures were inoculated every day using 1/200 volume of the previous culture.

\section{Animals}

Age-matched females, 6-8 weeks old BALB/C mice were purchased from Shanghai SLAC Laboratory Animal Co., Ltd. All mouse experiments were approved by the Institutional Animal Care and Use Committees of Zhejiang University and performed following the ethical guideline of the National Institutes of Health. No specific statistical consideration was taken in determining the sample size for the animal experiments.

\section{Bronchoalveolar Lavage Fluid (BALF) Collection}

Mice were euthanized, the trachea was exposed and a small hole was made for inserting a 20-gauge catheter. BALF was collected twice by instilling $0.6 \mathrm{~mL}$ of cold PBS. Then BALF was centrifugated at 5,000 r.p.m at $4^{\circ} \mathrm{C}$ for $10 \mathrm{~min}$ and the supernatant was used to quantify the level of lysostaphin, and the cell pellet was used for flow cytometry assay and inflammatory cell differentiation.

\section{Flow Cytometry}

Neutrophils in BALF were measured by flow cytometry. Red blood cells in the cell pellet were lysed with RBC lysis buffer at RT for 5 min and blocked by RPMI 1640 cell culture media, then centrifugated, washed by PBS. The cell pellet was collected and incubated with antibody cocktails including CD11b-PerCp Cy 5.5 (M1/70, BioLegend) anti-Ly-6G-Alexa Fluro 488 (1A8, BioLegend) for 30 min at RT. Then washed twice and resuspended with PBS. Flow cytometry 
was performed on a CytoFLEX LX flow cytometer. And the data files were analyzed with FlowJo (version 1.8.0_144-b01).

\section{Histology}

Whole lungs were dissected from mice were fixed with $4 \%$ formaldehyde, and then embedded, sectioned, and stained with H\&E or Gram stain for bacteria. Proinflammatory cytokines in the lung were assayed by the immunohistochemistry process according to the standard protocols. The stained samples were imaged with Olympus VS120. The histological score of H\&E staining was blinded performed according to the degree of neutrophil infiltration, alveolar hemorrhage, capillary congestion, and thickness of alveolar wall or hyaloid membrane formation. Each index is on a scale of 0 to $4: 0=$ no injury; $1=$ up to $25 \%$ injury of the field; 2 $=$ up to $50 \%$ injury of the field; $3=$ up to $75 \%$ injury of the field; and $4=$ diffused injury. ${ }^{1-3}$ The quantification of immunohistochemical staining was performed with image pro plus version 6.0. To address the cellular composition of inflammation in mice with different treatments, the total cell counts in BALF were calculated after red-blood-cell lysis. Then $2 \times 10^{5}$ cells resuspended in $30 \mu \mathrm{L}$ PBS were separated and deposited on a slide by Cytospin4 Centrifuge (Thermo Scientific) at 1,500 r.p.m for $5 \mathrm{~min}$ and then stained with Giemsa regent (Zhuhai Baso Biotechnology Co. LTD) for cell differentiation, as previously reported. ${ }^{1}$

\section{Cytotoxicity In Vitro}

The cytotoxicity of the complex to cells was performed on Beas-2B and A549 cells using the CCK-8 kit. Cell lines were cultured in Dulbecco's modified Eagle's Medium (DMEM) supplemented with $10 \%$ fetal bovine serum (FBS), penicillin (100 units $/ \mathrm{mL}$ ), and streptomycin $(100 \mu \mathrm{g} / \mathrm{mL})$ at $37{ }^{\circ} \mathrm{C}$ with $5 \% \mathrm{CO}_{2} .5 \times 10^{5}$ cells were seeded into each well of a 96 well plate in $100 \mu \mathrm{L}$ of growth medium. After $16 \mathrm{~h}$ of growth, the medium was removed and cells were washed once with PBS, and then serially diluted MS, Ly, LyIR@MS in $100 \mu \mathrm{L}$ culture medium was added to per well respectively and incubation was continued for $24 \mathrm{~h}$. Then CCK-8 solution was added and incubated for $1.5 \mathrm{~h}$. The absorbance in $570 \mathrm{~nm}$ was recorded by the SpectraMax M5 plate reader. 


\section{Evaluation of In Vivo Safety}

Healthy mice were given $100 \mu \mathrm{L}$ LyIR@MS, Ly (1 mg/mL of lysostaphin), or PBS through i.v. injection. The change of body weight and mortality were supervised every 2 day. At day 3 and day 28 after treatment, blood was collected to perform blood routine and serum biochemical examinations, and the major organs were separated for histological examination and stained with H\&E.

\section{REFERENCES}

(1) Ouyang, W.; Liu, C.; Pan, Y.; Han, Y.; Yang, L.P.; Xia, J.; Xu, F. SHP2 Deficiency Promotes Staphylococcus aureus Pneumonia Following Influenza Infection. Cell Prolif. 2020, 53, e12721.

(2) Simons, R. K.; Maier, R. V.; Chi, E. Y. Pulmonary Effects of Continuous Endotoxin Infusion in the Rat. Circ. Shock 1991, 33, 233-243.

(3) Zhang, Y.; Xu, T.; Wu, B.; Chen, H.; Pan, Z.; Huang, Y.; Mei, L.; Dai, Y.; Liu, X.; Shan, X.; Liang, G. Targeting Myeloid Differentiation Protein 2 by the New Chalcone L2H21 Protects LPS-Induced Acute Lung Injury. J. Cell Mo.I Med. 2017, 21, 746-757. 

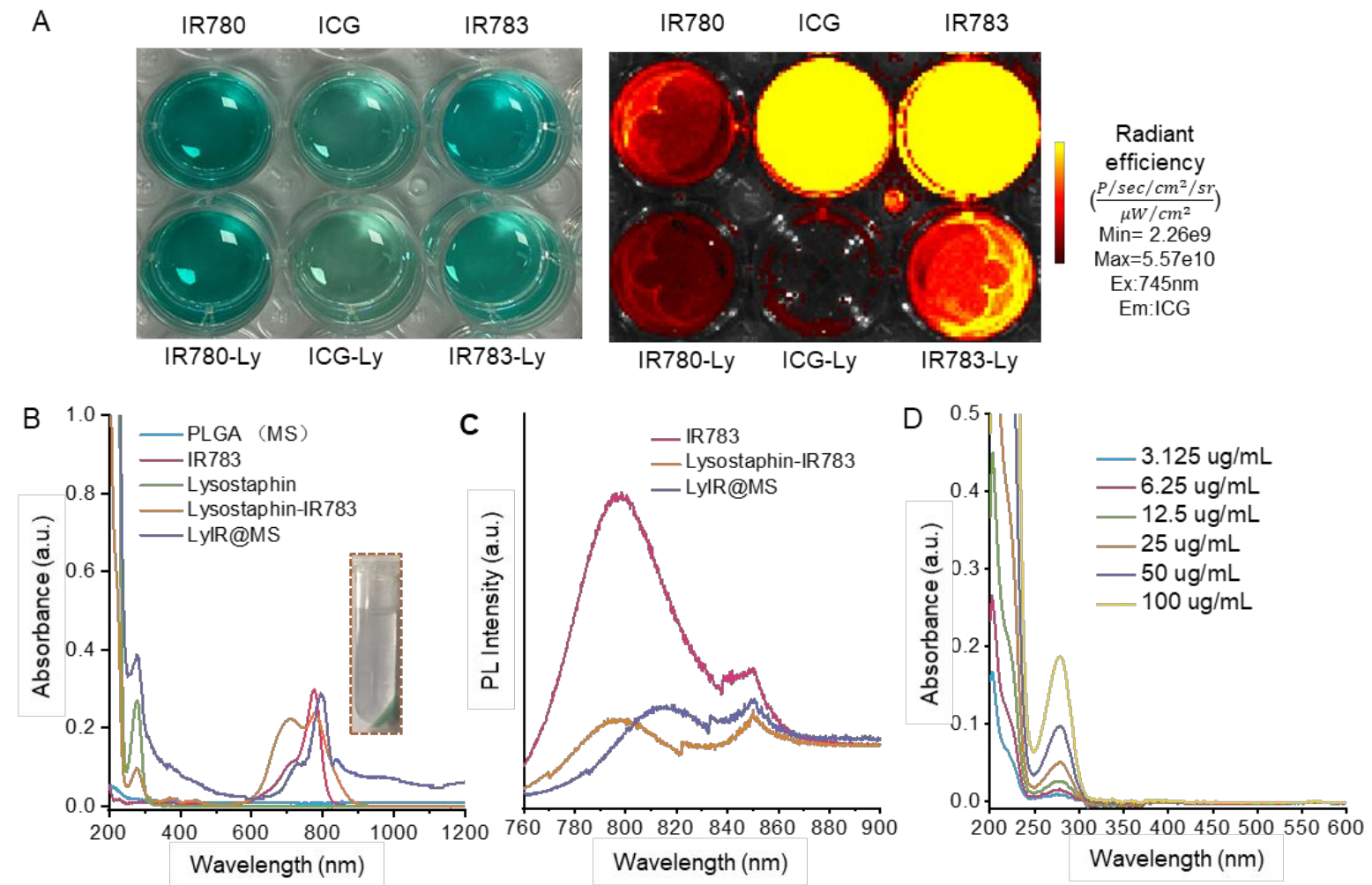

E

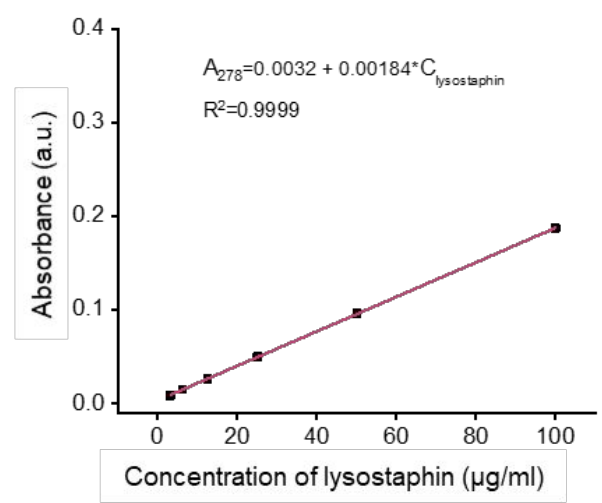

F

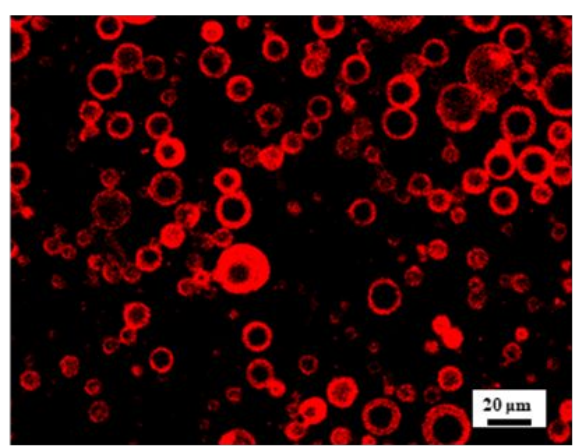

Supporting Figure 1. Design and characterization of hollow visualized LyIR@MS. (A) Photographs (left) and corresponding fluorescent images (right) of lysostaphin-dye complex, including FDA-approved Indocyanine Green (ICG) and its derivatives (IR780 and IR783). (B) Absorption spectra of PLGA microspheres (MS), IR783 dye, lysostaphin, lysostaphin-IR783 complex, and LyIR@MS. Insert photos are their precipitation after centrifugation (C) Emission spectra of IR783 dye, lysostaphin-IR783 complex, and LyIR@MS with excitation at $745 \mathrm{~nm}$. (D and E) Absorption spectra (D)of lysostaphin with a different concentration in DI water and standard curve $(E)$ with a characteristic absorption peak at $278 \mathrm{~nm}$. (F) Fluorescence microscopy image of cy5.5-staining LyIR@MS. 
A
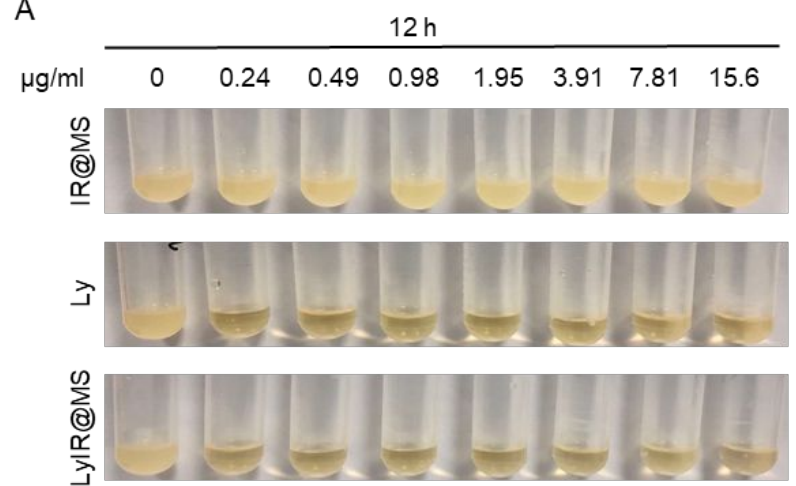

B

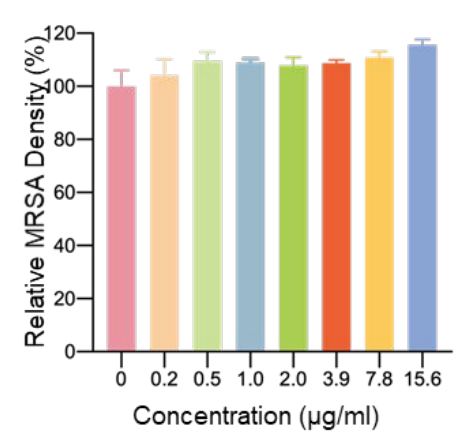

C

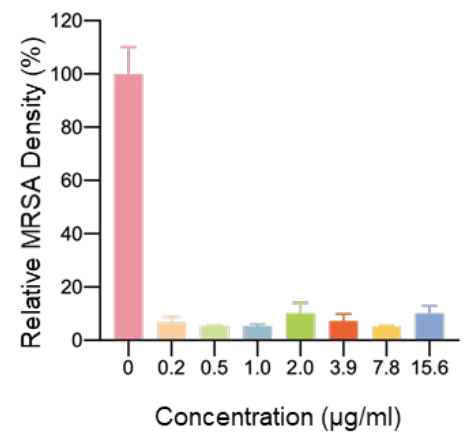

$24 \mathrm{~h}$
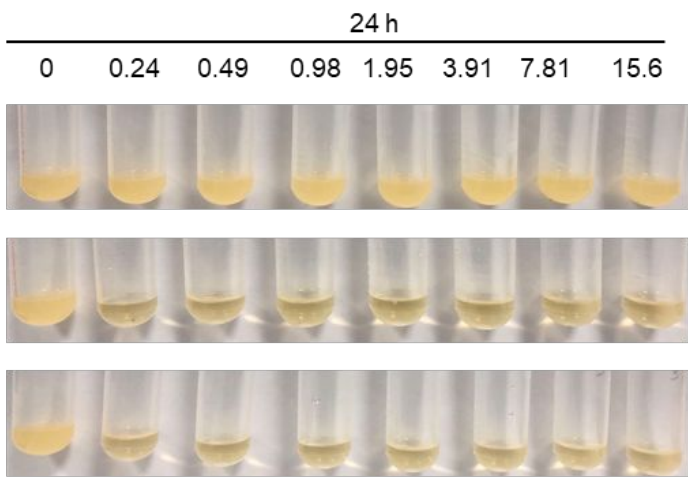

$\mathrm{D}$

Supporting Figure 2. Minimal inhibition concentration of LyIR@MS against MRSA. (A) Photographs of MRSA culture after $12 \mathrm{~h}$ and $24 \mathrm{~h}$ incubation with increasing concentrations of IR@MS, Ly, LyIR@MS. (B to D) The survival rate of MRSA at $24 \mathrm{~h}$ after coculture with indicated concentrations of IR@MS (B), Ly (C), and LyIR@MS (D). All data are showed as mean $\pm S D$. 
A

$\mathrm{Oh}$

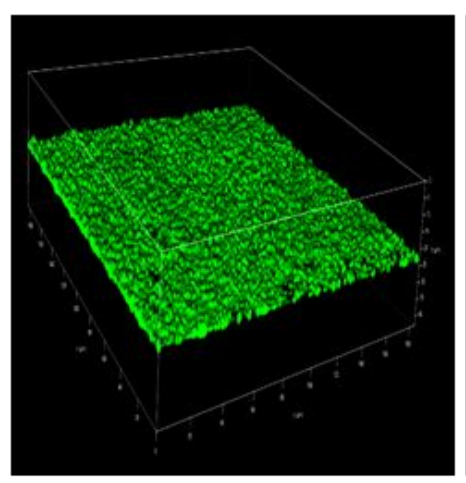

B

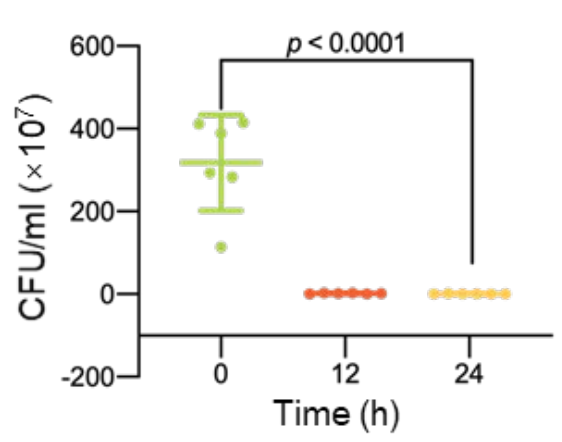

$12 \mathrm{~h}$

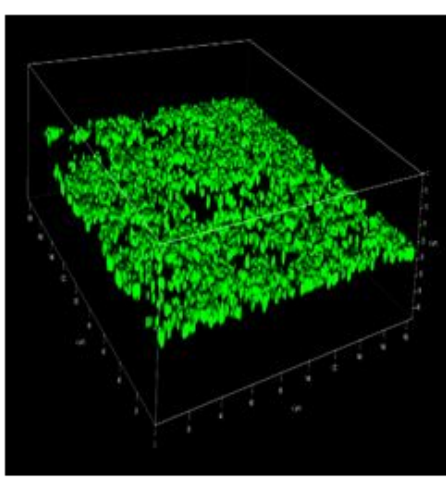

C
$24 \mathrm{~h}$

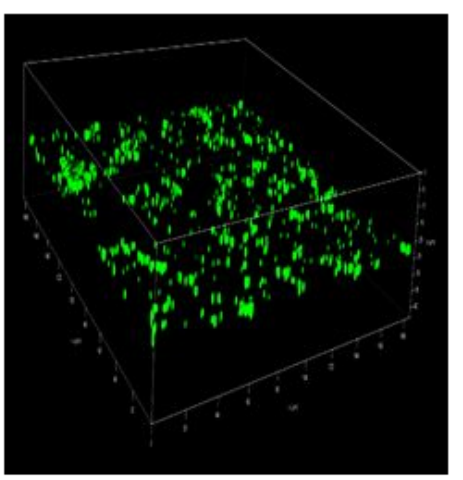

$12 \mathrm{~h}$

$24 \mathrm{~h}$

Supporting Figure 3. Anti-biofilm activity of LyIR@MS in a time-dependent manner. (A) CLSM images of biofilms treated with LyIR@MS (500 $\mu \mathrm{g} / \mathrm{mL}$ of lysostaphin) for the indicated time. ( $B$ and $C$ ) Time-dependent killing effect of LyIR@MS against MRSA formed biofilms showed by CFU counting (B) and agar plate assay (C). Data in (B) are expressed as mean \pm SEM. 

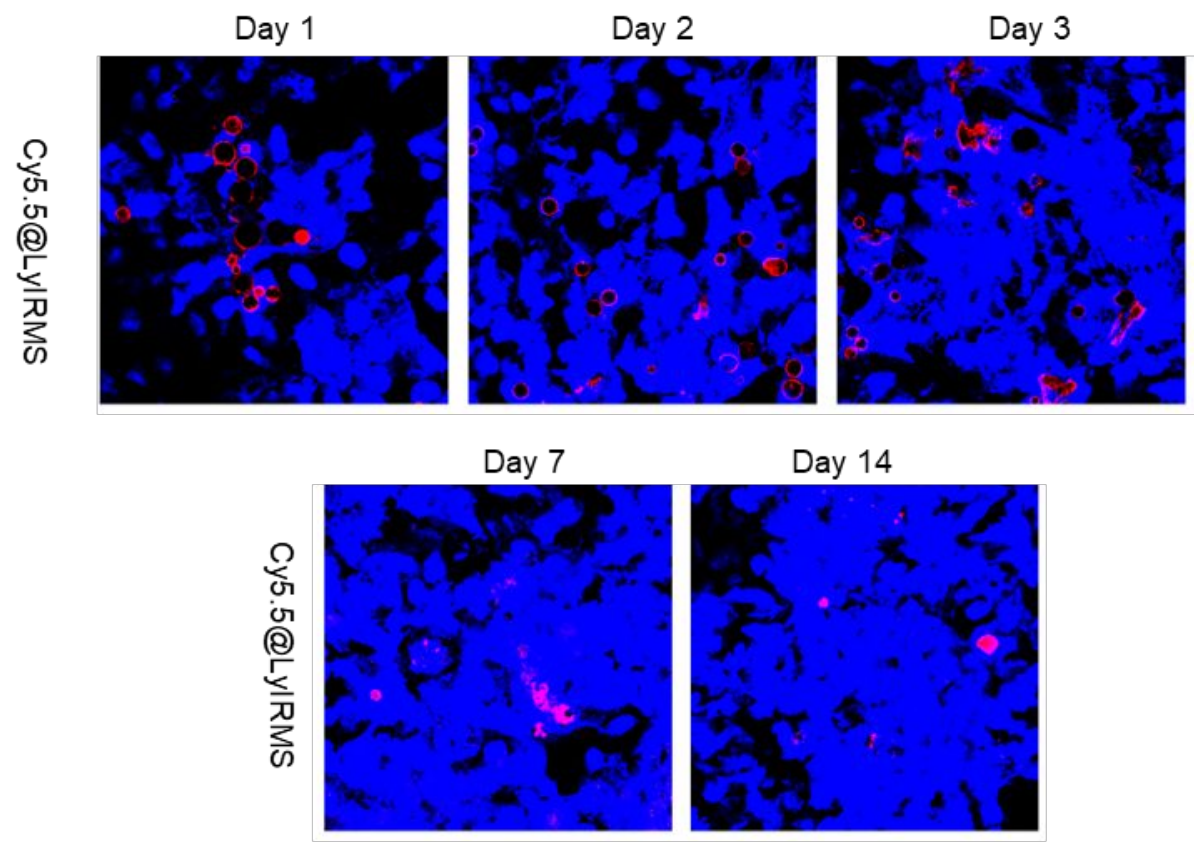

Supporting Figure 4. Confocal laser scanning microscopy images of lung tissues at different times after intravenous injection of cy5.5-staining LyIR@MS. The blue fluorescence represents the nucleus stained by DAPI, and the red represents the gradually degraded microspheres. 


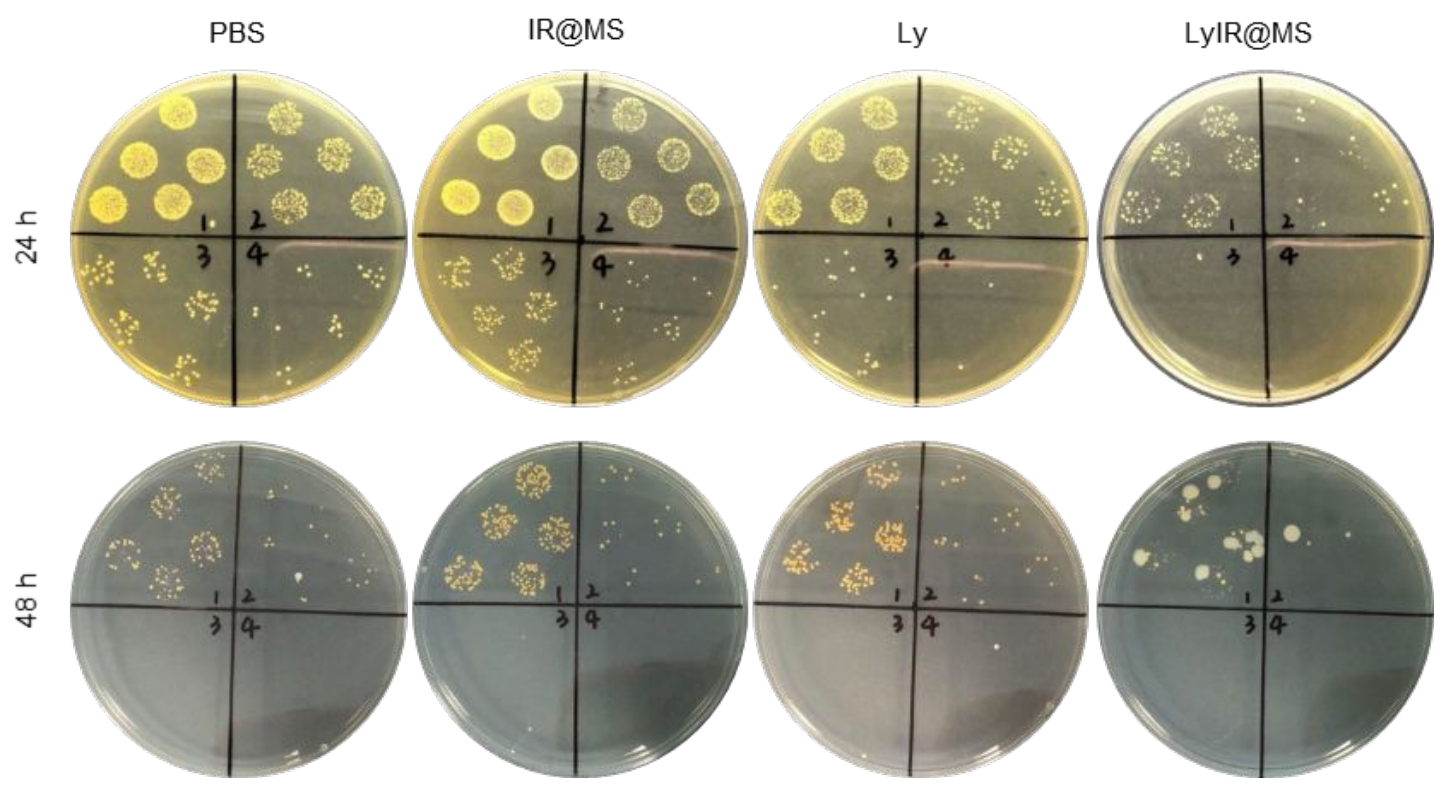

Supporting Figure 5. Bacterial load in the lung of mice in the post-exposure treatment study. Agar plate images of MRSA in lung homogenates of acute pneumonia mice after treatment with PBS, IR@MS, Ly, and LyIR@MS (1 mg/mL of lysostaphin) at $24 \mathrm{~h}$ and 48 h post-infection. 
A

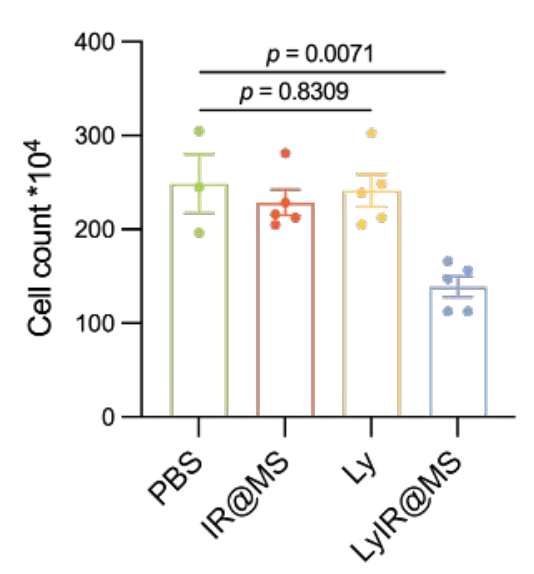

C

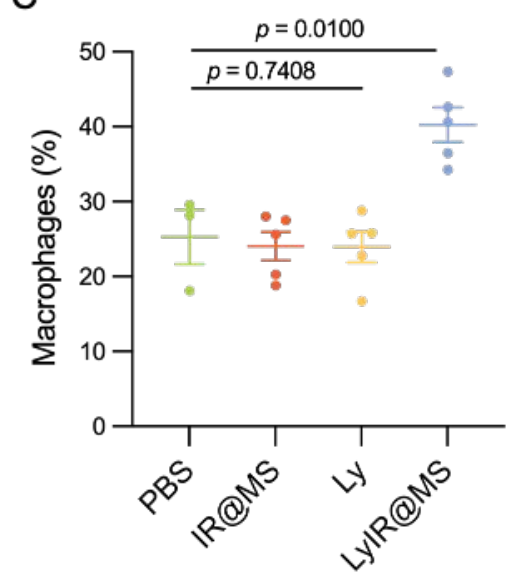

B

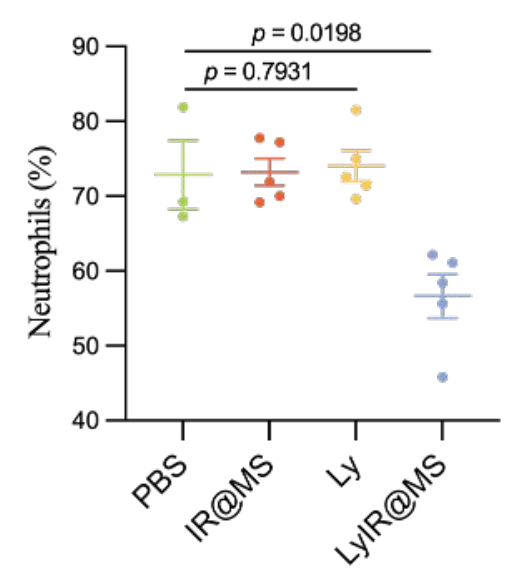

D

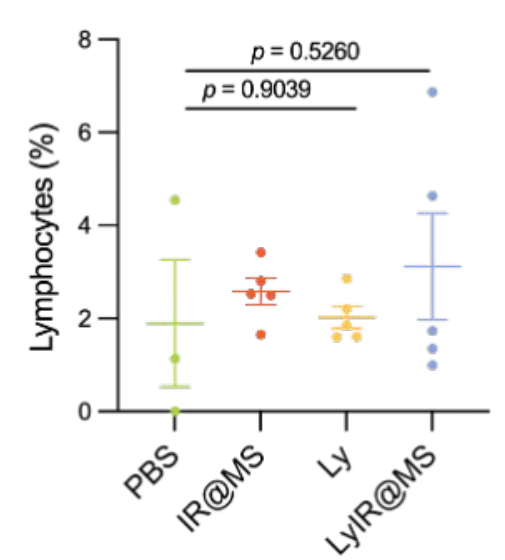

Supporting Figure 6. Analysis of cellular composition in BALF $24 \mathrm{~h}$ after infection. (A) Total cell counts in the BALF. (B to D) Proportion of neutrophils (B), macrophages (C) and lymphocytes $(D)$ in the BALF from different group. Data are presented as mean \pm SEM. 
A
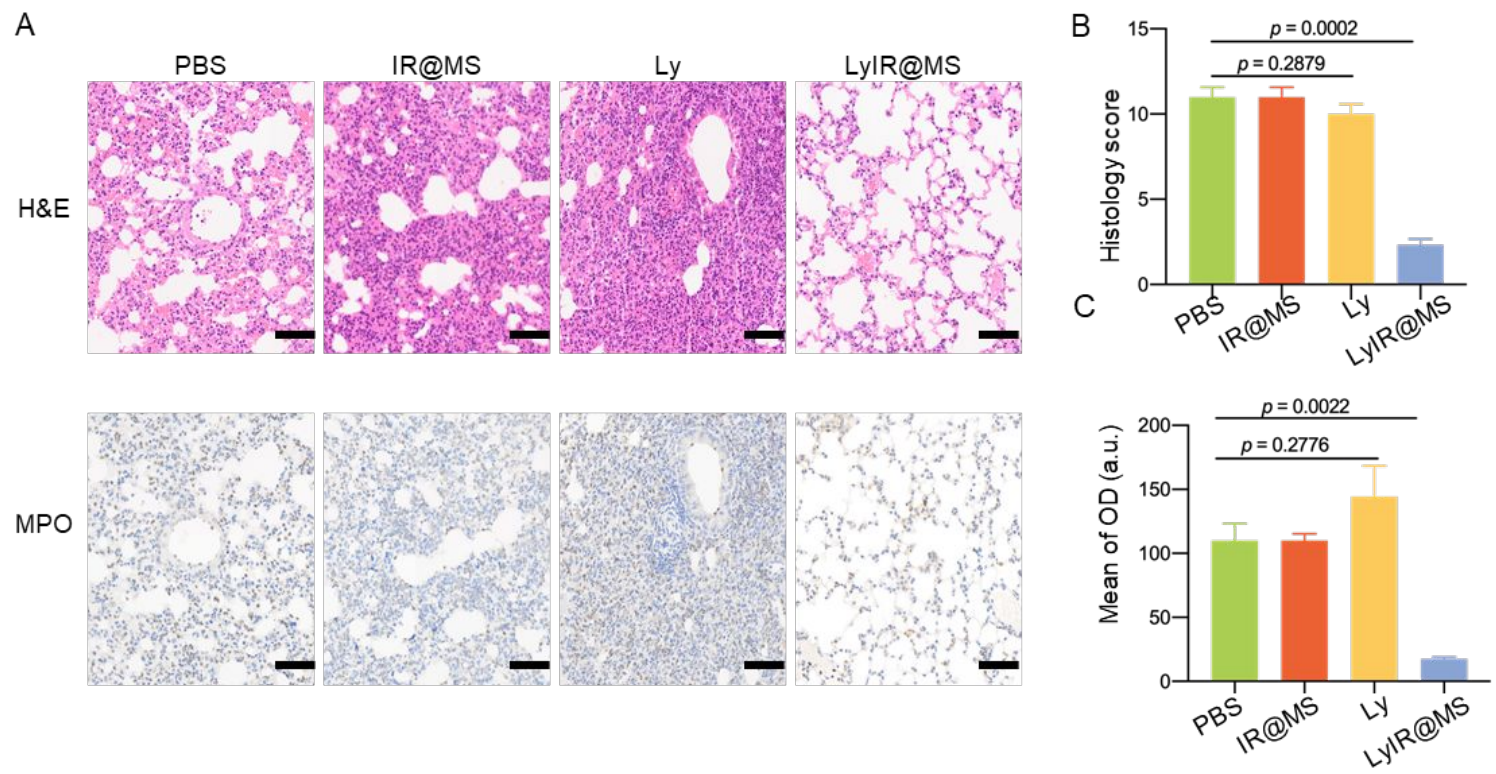

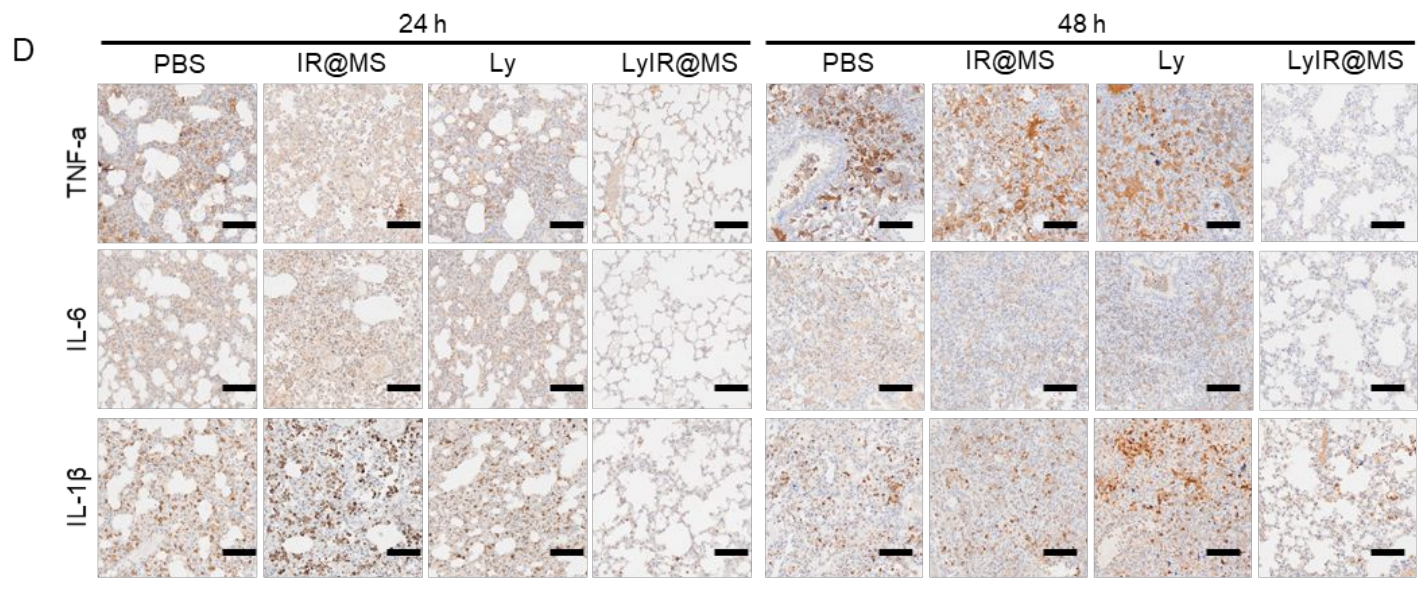

E

$\mathrm{F}$
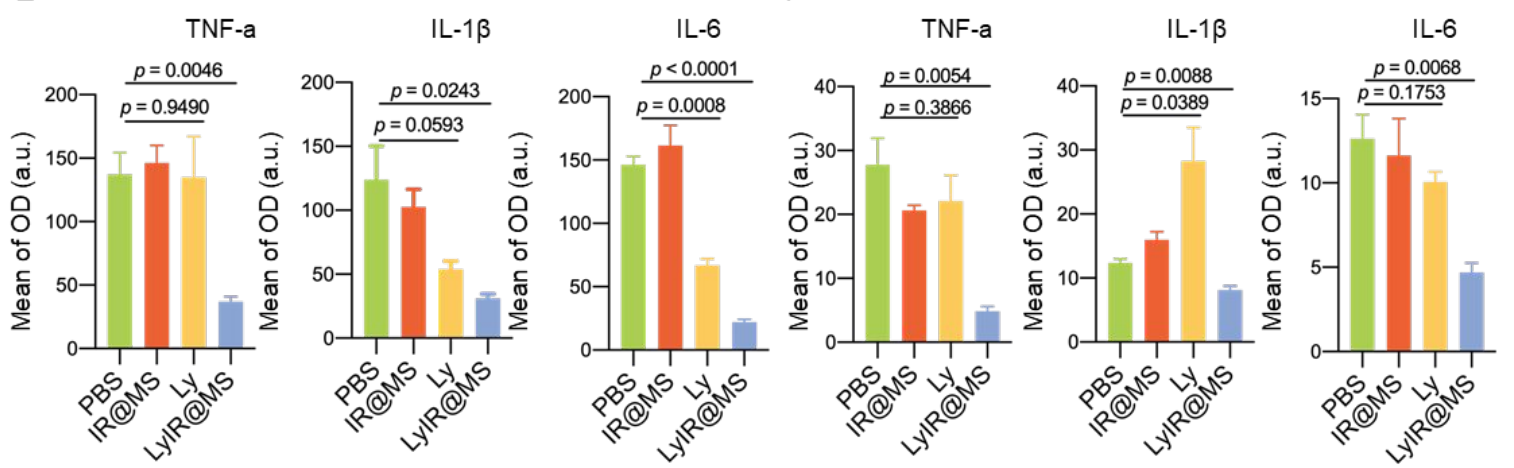

Supporting Figure 7. H\&E and immunohistochemical staining of lung sections in the post-exposure treatment study. (A to $C$ ) Representative images of H\&E stained and immunohistochemically stained (MPO) lung sections (A) from PBS, IR@MS, Ly, LyIR@MS (1 $\mathrm{mg} / \mathrm{mL}$ of lysostaphin) treated mice, quantification of H\&E score (B) and MPO expression (C) at $48 \mathrm{~h}$ post-infection. Scale bar, $50 \mu \mathrm{m}$. (D and F) Images (D) and quantification (E and F) of immunohistochemical staining of TNF- $\alpha$, IL- 6 , and IL-1 $\beta$ in lung sections of mice treated with PBS, IR@MS, Ly, and LyIR@MS at 24 h (E) and 48 h (F) after infection. Scale bar, 25 $\mu \mathrm{m}$. All results are expressed as mean \pm SEM. 

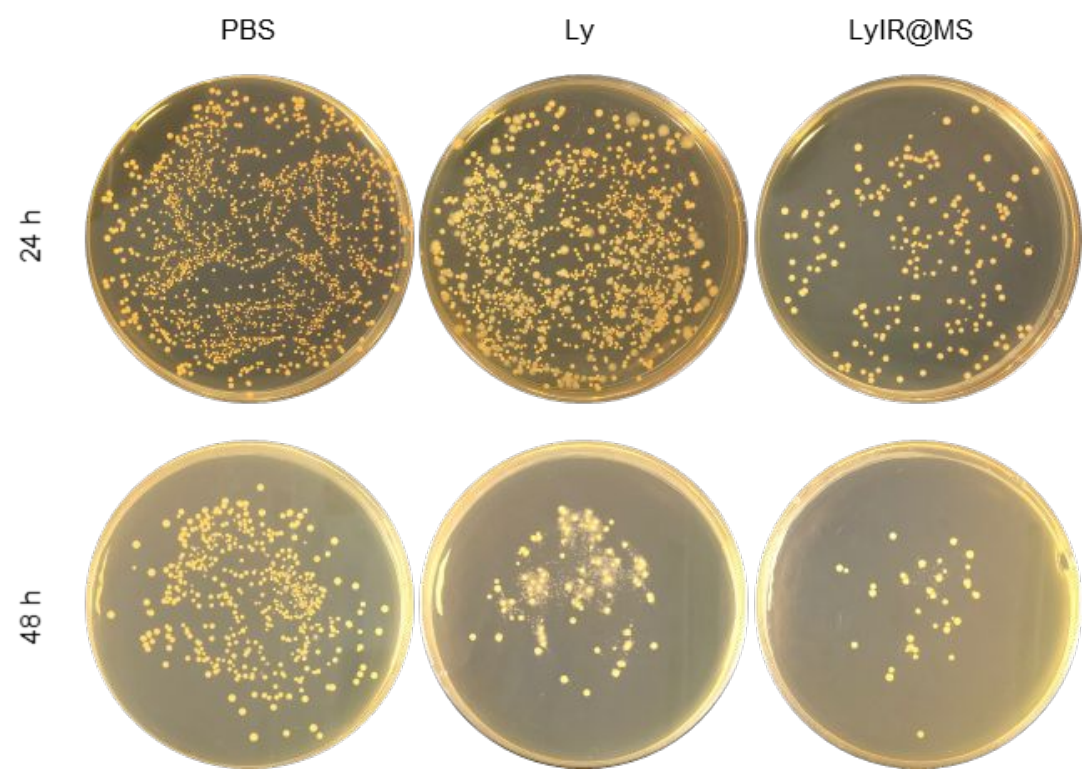

Supporting Figure 8. Bacterial load in the lung of mice in pre-exposure prophylaxis study. Agar plate images of MRSA in lung homogenates of acute pneumonia mice pre-exposure prophylaxis with PBS, IR@MS, Ly, and LyIR@MS (1 mg/mL of lysostaphin), at $24 \mathrm{~h}$ and $48 \mathrm{~h}$ post-infection. 